\title{
Arbeitskräftemobilität in der EU im Angesicht der Krise: Gewinner und Verlierer
}

Ist freie Arbeitskräftemobilität in der EU ein Positivsummenspiel, wie häufig von der EU-Kommission dargestellt, oder gibt es Gewinner und Verlierer? Die Krise hat einige der Schwachstellen der Arbeitskräftemobilität in einem vereinigten, aber diversen Europa sichtbar gemacht. Zumindest in Teilen hat kurzfristige Arbeitsmigration in den meisten EU15-Empfängerländern als Arbeitsmarktpuffer in der Krise gewirkt, und Migranten aus EU10-Ländern waren allgemein stärker von Arbeitslosigkeit betroffen als Einheimische. Einer unser wichtigsten Befunde aber ist die unterwertige Beschäftigung der EU10Migranten, wobei ganz Europa als Verlierer zählen muss. Diese Verschwendung von Humankapital verweist auf eine der größten Herausforderungen der Arbeitskräftemobilität innerhalb der EU. ${ }^{1}$

JANINE LESCHKE, BELA GALGÓCZI

\section{Einleitung}

Das Recht auf Freizügigkeit für Bürger und Arbeitnehmer aus EU-Mitgliedstaaten gilt als Kernwert der Europäischen Union und ist eine ihrer tragenden Säulen. Ein Jahrzehnt nach der Osterweiterung von 2004 und im sechsten Jahr der Wirtschaftskrise analysieren wir die potenzielle Bedeutung der Arbeitskräftemobilität in einer größeren und diverseren EU unter den turbulenten Bedingungen der Krise und versuchen, Gewinner und Verlierer auszumachen. Wir vergleichen, wie sich die Beschäftigungssituation von EU8 $-{ }^{2}$ und EU2-Migranten (Rumänien und Bulgarien) sowie einheimischen Arbeitnehmern in ausgewählten EU15-Ländern (EU-Mitgliedstaaten vor 2004) im Laufe der Krise entwickelt hat.

Durch die Wirtschaftskrise erhielt die Debatte über die Arbeitskräftemobilität innerhalb der EU neuen Auftrieb. Dabei vertritt die Europäische Kommission, beispielsweise im „Aktionsplan für berufliche Mobilität“ (European Commission 2010), die Ansicht, die Mobilität der Arbeitnehmer spiele für ein wirksameres Funktionieren der europäischen Arbeitsmärkte und eine erhöhte Arbeitsmarktflexibilität eine ausschlaggebende Rolle. Durch vermehrte Arbeitnehmermobilität ließe sich dank einer besseren Abstimmung zwischen den Kompetenzen der Arbeitsuchenden und dem Stellenangebot eine optimierte Verteilung der Arbeit inner- halb der EU erzielen und somit das gemeinschaftliche Bruttoinlandsprodukt (BIP) steigern. Es wird argumentiert, durch die Arbeitskräftemigration ließen sich auf den Arbeitsmärkten der Empfängerländer Qualifikationsengpässe beheben (European Commission 2011a). Die Überlegung, inwieweit Wanderarbeitnehmer die mitgebrachten Qualifikationen tatsächlich einsetzen können, wird dagegen weit seltener angesprochen.

Um den Rahmen der Untersuchung abzustecken, wollen wir uns im ersten Teil dieses Artikels zunächst mit der Arbeitnehmerfreizügigkeit und ihren Herausforderungen befassen. Im zweiten Teil beschreiben wir anhand aktueller Daten, die wir spezifischen Auszügen aus der aggregierten Europäischen Arbeitskräftestichprobe entnehmen, europaweite Trends in der grenzüberschreitenden Arbeitskräftemobilität während der Krisenjahre. Insbesondere untersuchen wir dabei die Entwicklung des Beschäftigtenbestandes und der Arbeitslosenraten von EU8- und EU2-Migranten

1 Übersetzung aus dem Englischen von Lisa Eskuche.

2 EU8 bezeichnet die im Mai 2004 beigetretenen mittel- und osteuropäischen Länder. Zypern und Malta traten der Union ebenfalls 2004 bei, wurden an dieser Stelle jedoch ausgeklammert, da sie nicht von Einschränkungen der Arbeitskräftefreizügigkeit betroffen waren. Wenn wir im Folgenden die Bezeichnung EU10 verwenden, meinen wir damit sowohl die EU8- als auch die EU2-Länder. 
im Vergleich zu einheimischen Arbeitnehmern in ausgewählten EU15-Ländern. In einem weiteren Schritt beurteilen wir das Missverhältnis zwischen Qualifikationsniveau und Beschäftigung, indem wir für EU10-Wanderarbeitnehmer die Daten der Europäischen Arbeitskräftestichprobe zu den Qualifikationen und der branchenspezifischen Verteilung der Arbeitnehmer auswerten. Unser Artikel schließt mit einer Kosten-Nutzen-Abwägung der grenzüberschreitenden intra-EU-Arbeitskräftemobilität aus Sicht der Herkunfts- und der Aufnahmeländer und versucht damit, die Frage nach den potenziellen „Verlierern“ und „Gewinnern“ der Arbeitskräftemigration zu beantworten.

\section{Arbeitnehmerfreizügigkeit - ein Kernwert der EU}

Mit der EU-Erweiterung um acht mittel- und osteuropäische Länder (EU8) im Mai 2004 sowie dem anschließenden Beitritt Rumäniens und Bulgariens im Januar 2007 (EU2) wurde in der Geschichte der europäischen Integration ein neues Kapitel aufgeschlagen. Eine wichtige Folge der Erweiterungsrunden war die Ausdehnung von Freizügigkeit und freiem Waren-, Dienstleistungs- und Kapitalverkehr nach Mittel- und Osteuropa. In den Beitrittsländern wurde das Recht auf „Freizügigkeit“ einhellig als ein Grundrecht empfunden. In der EU15 hingegen gingen der Erweiterung intensive und teilweise kontrovers geführte Diskussionen über den Umfang der zu erwartenden Zuwanderung voraus. Zur Debatte stand auch die Frage, ob die bisherigen Mitgliedstaaten ihre Arbeitsmärkte mit sofortiger Wirkung vollumfänglich für die neuen Mitgliedsländer öffnen und damit eine uneingeschränkte Arbeitnehmerfreizügigkeit ermöglichen sollten, oder ob die bis dahin bestehenden Einschränkungen für die Dauer der vorgesehenen Übergangsperiode beibehalten werden sollten. Die öffentliche Debatte spielte sich vor dem Hintergrund gewaltiger Unterschiede - beispielsweise beim Lohnniveau - und teilweise hoher Arbeitslosigkeit ab und kreiste unter anderem um die Rolle von Arbeitsmigranten in hoch entwickelten Volkswirtschaften und Gesellschaften. Zumindest in einigen Ländern war sie von zunehmend populistischen und fremdenfeindlichen Strömungen begleitet. Stimmungsmacher warnten vor einem Massenzustrom von Arbeitsuchenden und angeblichen Sozialbetrügern und sorgten damit in weiten, bereits durch Globalisierung und Abwanderung von Arbeitsplätzen verängstigten Teilen der Bevölkerung für weitere Verunsicherung. In bestimmten Ländern - darunter die Niederlande und Dänemark - hatten diese Diskussionen eine Umkehr der ursprünglichen Entscheidung gegen Übergangsregelungen zur Folge (Kvist 2004). Im Endeffekt verzichteten 2004 nur drei Länder (Großbritannien, Irland und Schweden) auf temporäre Regelungen; alle anderen schränkten das Recht der EU8-Bürger, in den jeweiligen EU15-
Ländern zu arbeiten, für einen Zeitraum von bis zu sieben Jahren mehr oder weniger stark ein. Im Laufe der letzten zehn Jahre wurden die EU15-Arbeitsmärkte sukzessiv und zeitversetzt für die Bürger aus EU8-Ländern geöffnet; lediglich Deutschland und Österreich schöpften die siebenjährige Übergangsfrist voll aus. Für Arbeitnehmer aus Bulgarien und Rumänien (EU2) hielten dagegen neun Länder an der vollen Übergangsfrist bis 1. Januar 2014 fest, wobei die Verschlechterung der Wirtschaftsaussichten mit eine Rolle spielte. Viele der Länder, die gegenüber den EU2 Übergangsregelungen anwendeten, waren von der Wirtschaftskrise jedoch vergleichsweise wenig betroffen. ${ }^{3}$

Geschichtlich gesehen stellt die seit 2004 bestehende Arbeitnehmerfreizügigkeit in mehrfacher Hinsicht ein neuartiges Phänomen dar. Erstens handelt es sich dabei um einen vielschichtigen Prozess, bei dem unterschiedliche Arten von Arbeitskräftemobilität parallel zueinander in einem sich rapide verändernden Umfeld existieren - ein Umstand, der durch die Krise in jüngerer Zeit zusätzlich an Bedeutung gewann. Zu den Erscheinungsformen der grenzüberschreitenden Arbeitskräftemobilität zählen Grenzgänger ebenso wie kurzfristige, „zirkuläre“ und dauerhaftere Migration; aber auch funktional mit Migration gleichzusetzende Formen der Arbeitsmobilität wie die Entsendung von Arbeitskräften (IDEA Consult ECORYS Netherlands 2011) und (schein-)selbstständige Erwerbstätigkeit im Rahmen des freien Dienstleistungsverkehrs (Cremers 2011) spielen eine wichtige Rolle. Neu ist auch, dass Migranten aus Niedriglohnländern sowohl absolut gesehen als auch in Relation zu den einheimischen Arbeitnehmern der Aufnahmeländer ein vergleichsweise hohes Bildungsniveau besitzen (siehe Abschnitt 4).

Die historischen Wanderungsmuster und Arbeitnehmerbewegungen aus der Zeit vor der EU-Erweiterung verlagerten sich in Richtung auf diejenigen EU15-Länder, die ihre Arbeitsmärkte sofort nach der Erweiterung öffneten und in denen zur selben Zeit eine hohe Nachfrage nach Arbeitskräften bestand (Galgóczi et al. 2009; Kahanec/Zimmermann 2010). Infolgedessen waren in den meisten Ländern die Auswirkungen des grenzüberschreitenden Migrationsgeschehens während der ersten Jahre nach der Erweiterung deutlich weniger spürbar als erwartet; anderenorts - vor allem in Großbritannien und Irland sowie, in Bezug auf EU2-Migranten, in Italien und Spanien (Holland et al. 2011) - wanderten jedoch deutlich mehr Personen zu als vorhergesagt (eine kritische Bestandsaufnahme findet sich bei Dustmann et al. 2003; Heinz/Ward-Warmedinger 2006).

3 Folgende Länder machten gegenüber Bulgarien und Rumänien von der gesamten siebenjährigen Übergangsfrist Gebrauch: Belgien, Deutschland, Frankreich, Luxemburg, Malta, Niederlande, Österreich und Großbritannien (European Commission 2011b). 


\section{Auswirkungen der Wirtschaftskrise auf die grenzüberschreitende Arbeitskräftemobilität}

In diesem Abschnitt bieten wir einen Überblick über die wichtigsten Trends der grenzüberschreitenden Ost-WestArbeitskräftemobilität während der Krise. Dabei betrachten wir vor allem Veränderungen des Beschäftigtenbestands aus EU10-Ländern in EU15-Arbeitsmärkten sowie die Entwicklung der Arbeitslosenquoten für einheimische Arbeitnehmer und für Migranten aus der EU10.

Historisch betrachtet, wirken sich starke Konjunkturabschwünge auf die Wanderungsbilanz und besonders auf die Arbeitsmigration kurzfristig negativ aus; langfristig dagegen lässt sich im Normalfall keine Auswirkung auf Migrationstrends feststellen (OECD 2009). Entsprechend erwartete das European Integration Consortium (2009) aufgrund der Finanzkrise eine vorübergehende Dämpfung der Zuwanderung, da das Migrationsgeschehen weitgehend von den Beschäftigungsaussichten in den Aufnahmeländern bestimmt wird und ausländische Arbeitnehmer in Phasen des Konjunkturrückgangs überproportional von Stellenkürzungen betroffen sind. Wirtschaftsschocks wirken sich auf Migranten also besonders stark aus. Arbeitsmigranten sind überwiegend in sehr konjunkturanfälligen Bereichen beschäftigt, wie Industrie oder Hotel- und Gaststättengewerbe (siehe Abschnitt 4), und haben häufiger als einheimische Arbeitnehmer kurzfristige Arbeitsverträge. Hinzu kommen eine im Durchschnitt kürzere Betriebszugehörigkeit („Last in - First out“-Regel) sowie das Risiko von Benachteiligungen bei Einstellungen und betriebsbedingten Kündigungen (vergleiche OECD 2009; OECD 2013, Kapitel 4). Diese Aussagen decken sich mit unserem Befund in Galgóczi et al. (2009), dass vor allem die Nachfrage nach Arbeitskräften in den Aufnahmeländern das Wanderungsgeschehen bestimmt (siehe auch OECD 2012). Ahearne et al. (2009) konzentrieren sich in ihren Simulationen auf die Arbeitsmarktsituation in den Herkunftsländern und ihre Wirkung als Push-Faktor. In den vier am wenigsten von der Krise betroffenen Ländern - Tschechien, Polen, Slowakei und Slowenien - wird davon ausgegangen, dass die Abwanderung etwas geringer ausfallen wird als es ohne die Krise der Fall gewesen wäre. Für krisengebeutelte Länder wie die baltischen Staaten dagegen wird angesichts der Verschlechterung der Wirtschaftslage im Vergleich zur EU15 erwartet, dass die Auswanderung nach einem kurzen krisenbedingten Rückgang wieder rapide ansteigen wird.

Im folgenden Abschnitt beleuchten wir jüngste Entwicklungen im Bereich der Arbeitskräftemobilität innerhalb der EU sowie die Auswirkungen der Krise auf den Arbeitsmarkt; wir stützen uns dabei auf aktuelle aggregierte Daten der Europäischen Arbeitskräftestichprobe (AKE). Mangels aussagekräftiger Vergleichsdaten zu den Wanderungsströmen basieren die Informationen, nach Staatszugehörigkeiten aufgegliedert, auf Migrantenpopulationen und Beschäftigtenbeständen. Die Daten geben zum überwiegenden Teil die Situation in ausgewählten Aufnahmeländern wieder. ${ }^{4}$

\subsection{Haupttrends der Arbeitskräftemobilität in der EU unter besonderer Berücksichtigung des Krisenzeitraums}

Klammert man eine Minderheit von EU15-Ländern aus, in denen der Anteil von EU8- und EU2-Arbeitnehmern an der gesamten Erwerbsbevölkerung 2013 über $2 \%$ betrug (Irland, Italien, Österreich, Großbritannien, Spanien und Luxemburg), ist aus der Sicht der Aufnahmeländer die EUBinnenmobilität immer noch relativ gering. Dies gilt besonders für Frankreich, Portugal und Schweden (für ausgewählte Länder siehe Tabelle 1). Aus der Perspektive der Herkunftsländer ist das Ausmaß der Abwanderung dagegen problematisch, insbesondere in Anbetracht des niedrigen Alters und hohen Qualifikationsniveaus der abwandernden Arbeitnehmer. Im Zeitraum 2004 - 2009 lag die Abwanderung aus EU8-Ländern bei -1,8 \%, die aus EU2-Ländern bei $-6,3 \%$, wobei die baltischen Staaten, Polen, Bulgarien und insbesondere Rumänien die höchsten Auswanderungsquoten aufwiesen (Holland et al. 2011; Ambrosini et al. 2011; Dølvik/Eldring 2008).

Abbildungen 1 und 2 zeigen allgemeine Trends der OstWest-Arbeitsmigration seit der EU-Erweiterung und veranschaulichen die Entwicklung der Bestände getrennt nach der Herkunft der Beschäftigten aus EU8 und EU2. Für die beiden Aufnahmeländer, die ihren Arbeitsmarkt zum Zeitpunkt der Erweiterung öffneten, als die Bedingungen für die Eingliederung von Migranten in die jeweiligen Arbeitsmärkte vergleichsweise günstig standen - Großbritannien und Irland -, weist Abbildung 1 einen merklichen Anstieg der Anzahl von Beschäftigten aus EU8-Ländern im Zeitraum unmittelbar nach der Erweiterung aus. Der negative Einfluss der Krise auf die Arbeitskräftemigration aus der EU8 ist ab 2008 vor allem im hart getroffenen Irland deutlich zu erkennen: Die Anzahl der EU8-Beschäftigten, die dort 2008 mit 152.000 ihren Höchststand erreicht hatte, sank 2013 auf 118.000. In Großbritannien stagnierte die Anzahl von Beschäftigten aus EU8 Ländern im Zeitraum 2008 - 2009, zog aber ab 2009 wieder an.

In Deutschland, einem traditionellen Zielland für Migranten aus dem mittel- und osteuropäischen (MOE) Raum, galt die Frist für Übergangsregelungen bis Mai 2011. Bis 2010 war dort eine stetige, aber vergleichsweise langsame

4 Die Länderauswahl richtet sich nach der Größe der Aufnahmeländer (z. B. wurde Frankreich ungeachtet der relativ geringen Zuwanderung aus der EU10 aufgenommen), der Zahl der Zuwanderer aus EU10-Ländern (deshalb z. B. Einschluss von Österreich und Irland) und einer Abdeckung der unterschiedlichen Wohlfahrtsstaattypen (Einschluss Dänemarks). 
Zunahme der Beschäftigten aus EU8-Ländern zu beobachten. Ab 2010 beschleunigt sich der Anstieg, was sich sowohl mit dem Ende der Übergangsfrist als auch der vergleichsweise positiven Arbeitsmarktentwicklung jüngerer Zeit in Zusammenhang bringen lässt (Abbildung 1). Österreich, aufgrund seiner geografischen Lage und seiner günstigen Wirtschaftsbedingungen auch ein traditionelles Zielland für Arbeitnehmer aus MOE-Staaten, weist bis zum Auslaufen der Übergangsregelungen 2011 eine langsame Zunahme der Beschäftigten aus EU8-Ländern auf und ab diesem Zeitpunkt ebenfalls eine merkliche Beschleunigung.

Wie aus Abbildung 2 und Tabelle 1 ersichtlich, nahm auch die Zuwanderung aus Bulgarien und Rumänien (EU2) deutlich zu. Absolut betrachtet waren Großbritannien und Deutschland die beliebtesten Zielländer für Zuwanderer aus der EU8, für Migranten aus EU2 dagegen Spanien und insbesondere Italien (Tabelle 1). Zudem bestanden die Übergangsregelungen für EU2-Zuwanderer in Großbritannien und Deutschland während der gesamten siebenjährigen Übergangsfrist. Spanien hob die Übergangsregelungen nach Ablauf der ersten Phase auf, führte sie aber für rumänische Arbeitnehmer Mitte 2011 wieder ein. Italien behielt die Übergangsregelungen bis zum Ende der zweiten Phase (Dezember
TABELLE 1

Anteile der EU8- und EU2-Bürger an der Gesamtbeschäftigung in ausgewählten EU15-Ländern, 2005* und 2013

Angaben in Prozent

\begin{tabular}{lllllll}
\hline & $\mathbf{2 0 0 5}$ & $\mathbf{2 0 1 3}$ & $\mathbf{2 0 0 5}$ & $\mathbf{2 0 1 3}$ \\
\hline & EU8 & EU8 & EU2 & EU2 \\
\hline GB & 0,56 & 2,44 & 0,07 & 0,43 \\
DE & 0,62 & 1,22 & 0,14 & 0,40 \\
IE & 5,53 & 6,72 & 0,26 & 0,51 \\
AT & 1,36 & 2,04 & 0,45 & 0,93 \\
IT & 0,13 & 0,36 & 0,75 & 3,16 \\
ES & 0,23 & 0,28 & 1,89 & 2,34 \\
FR & 0,07 & 0,12 & 0,02 & 0,11 \\
DK & 0,10 & 0,82 & 0,05 & 0,46 \\
EU27 & 0,28 & 0,83 & 0,32 & 0,74 \\
\hline
\end{tabular}

* Bei Irland Zahlen für 2006, da für 2005 nicht verfügbar.
ABB. 1

Arbeitnehmer aus EU8 in ausgewählten EU15-Ländern, 2005-2013

Angaben in Tsd., Beschäftigtenbestände
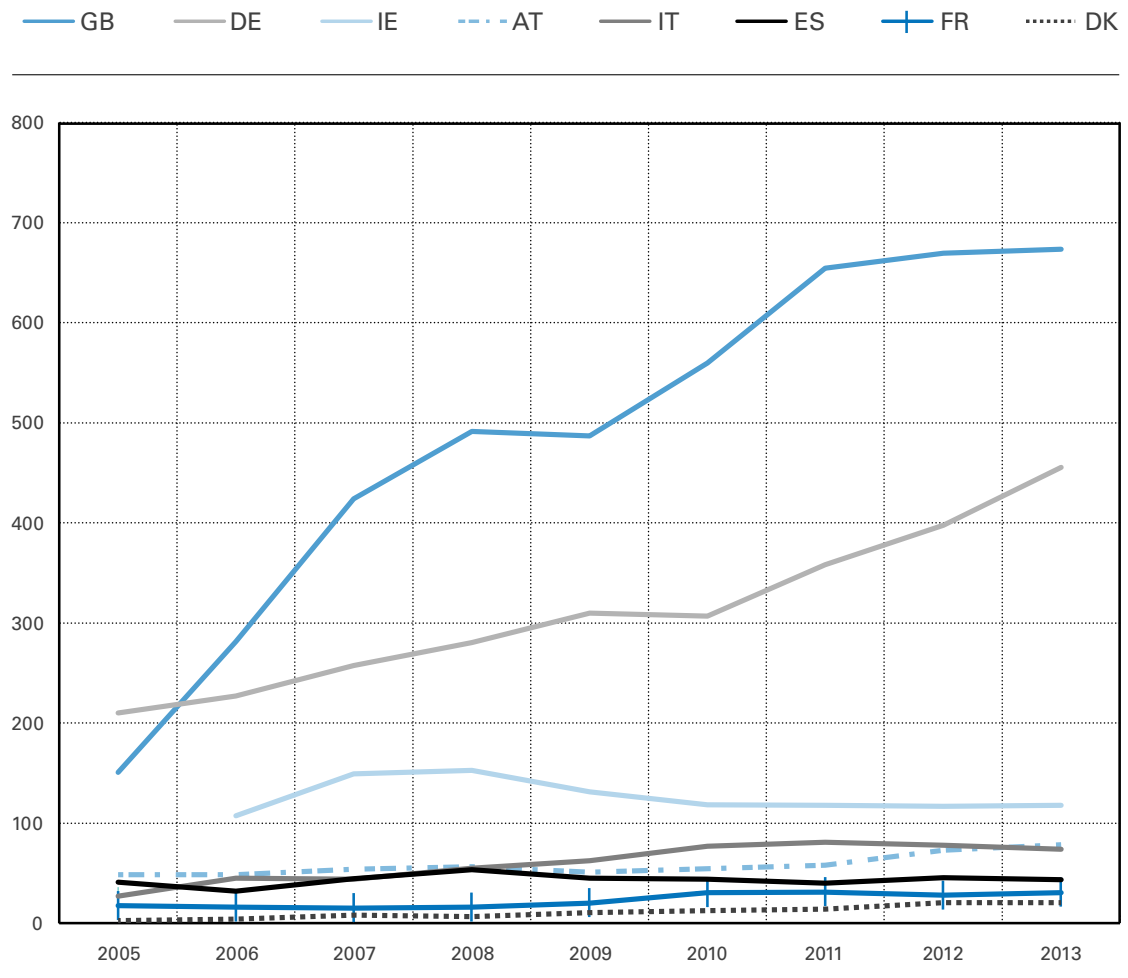

Quelle: Eurostat-Sonderauszüge aus aggregierten AKE-Daten, Version 28.08.2014. Alter: 15-64. Berechnung und Darstellung der Autoren.
2011) bei, verzichtete jedoch in speziellen, auf Migranten angewiesenen Sektoren (wie Landwirtschaft, häusliche Arbeit, Pflegedienste, Saisonarbeit) auf die Arbeitserlaubnispflicht. Eine bremsende Wirkung der Krise und ihrer Arbeitsmarktfolgen auf das rapide Anwachsen der Migrantenpopulation und der Beschäftigten aus EU2-Staaten lässt sich für Italien nicht beobachten. Im krisengezeichneten Spanien dagegen flachte die anfänglich starke Zunahme von Beschäftigten aus der EU2 entsprechend der Wirtschaftslage ab und war - bis zu einer leichten Erholung in allerjüngster Zeit - ab 2009 rückläufig (siehe auch die ausführlichere Analyse in Abschnitt 3). Der starke Anstieg der Zahl Beschäftigter aus EU2-Staaten in einer Reihe von EU15-Ländern muss unter anderem vor dem Hintergrund der gewaltigen wirtschaftlichen und sozialen Unterschiede (z. B. bei den Löhnen) zwischen Bulgarien und Rumänien auf der einen Seite und den EU15-Ländern auf der anderen gesehen werden.

Tabelle 1 liefert ergänzende Informationen, die nicht nur Trends illustrieren, sondern auch die relative Bedeutung der Zuwanderung aus EU2- und EU8-Ländern für bestimmte EU15-Arbeitsmärkte wiedergeben. Der Anteil der EU8Migranten an der Gesamtbeschäftigung liegt in Irland mit Abstand am höchsten, ist aber auch in Großbritannien und Österreich relativ hoch. Sehr gering ist der Anteil der EU8und EU2-Migranten dagegen nach wie vor in Frankreich. Dänemark bleibt hinsichtlich des Anteils von EU2-Migranten trotz sehr hoher relativer Zuwächse im Untersuchungszeitraum deutlich unter dem EU-Durchschnitt, während der Anteil von Arbeitnehmern aus EU8-Ländern knapp dem Durchschnitt entspricht. 
Auf die Arbeitsmärkte wirkte sich die Krise sowohl in den Aufnahmeländern als auch in den Herkunftsstaaten sehr unterschiedlich aus. In Polen, dem Land mit der höchsten absoluten Abwanderungsrate, nahm die Krise einen relativ glimpflichen Verlauf - Polen blieb als einziges Land von einem Produktionsschock verschont. Dagegen schnellte vor allem in den baltischen Staaten, insbesondere während der Anfangsphase der Krise, die Arbeitslosigkeit in die Höhe und war begleitet von einem erheblichen Beschäftigungsrückgang. Für Lettland und Estland befindet Hazans (2012), dass entsprechend der Wirtschaftslage Push-Faktoren zunehmend an Einfluss gewannen (vor allem der Faktor Arbeitslosigkeit, in Lettland aber auch allgemeine Unzufriedenheit) und dass in der Folgezeit die - von betrieblichen Kündigungen überproportional betroffene - Gruppe der geringqualifizierten Arbeitnehmer unter den Fortziehenden überrepräsentiert war. Im Fall Rumäniens bezeichnen Stan/Erne (2013) die Entwicklungsmuster der postkommunistischen Zeit als Auslöser der Auswanderungsschübe in den 1990er Jahren und nach der EU-Erweiterung 2007. Faktoren mit prägendem Einfluss auf das Migrationsgeschehen und die Wanderungsmuster rumänischer Bürger sind nach Meinung der Verfasser, erstens, die Aushebelung der Überlebensstrategien, durch die sich gewisse benachteiligte Bevölkerungsgruppen unter den Bedingungen der Subsistenzwirtschaft ein Auskommen sichern konnten, zweitens, die Rolle italienischrumänischer Kooperationsprojekte im Bereich der Bekleidungsindustrie sowie, drittens, der migrationsfördernde Effekt der in den 1990er Jahren entstandenen Migrantennetzwerke.

\subsection{Arbeitslosigkeit}

Bezüglich der unmittelbaren Arbeitsmarktfolgen der Krise ist festzustellen, dass Arbeitsmigranten aus EU10-Ländern in der Mehrzahl der EU15-Länder stärker betroffen waren als Einheimische und zumindest zum Teil als Arbeitsmarktpuffer dienten. Dies lässt sich beispielsweise anhand von Veränderungen der Arbeitslosenquoten für Einheimische und EU10-Migranten nachweisen.

So lässt Abbildung 3 für EU10-Migranten einen überproportional hohen Anstieg der Arbeitslosenquote insbesondere in Irland, Spanien (und Griechenland) erkennen, wobei zu beachten ist, dass die Arbeitslosenrate für Zuwanderer aus der EU10 in fast allen EU15-Ländern generell höher liegt als für Einheimische. Die größere Krisenanfälligkeit von Arbeitnehmern aus der EU10 ist bedingt durch die wesentlich höheren Beschäftigungsanteile von Bürgern aus diesen Ländern in Sektoren, in denen sich der Produktionseinbruch unverhältnismäßig stark bemerkbar machte (vgl. Abschnitt 4). Extrem hoch waren die Verluste beispielsweise im Baugewerbe, in dem besonders viele Arbeitnehmer aus der EU10 tätig sind, und wo während der Krise europaweit Millionen von Arbeitsplätzen abgebaut wurden.
ABB. 2

Arbeitnehmer aus EU2 in ausgewählten EU15-Ländern, 2005-2013

Angaben in Tsd., Beschäftigtenbestände
- DE
- IE $\quad-\cdots$ AT
- IT -ES $\longrightarrow$ FR
…... DK

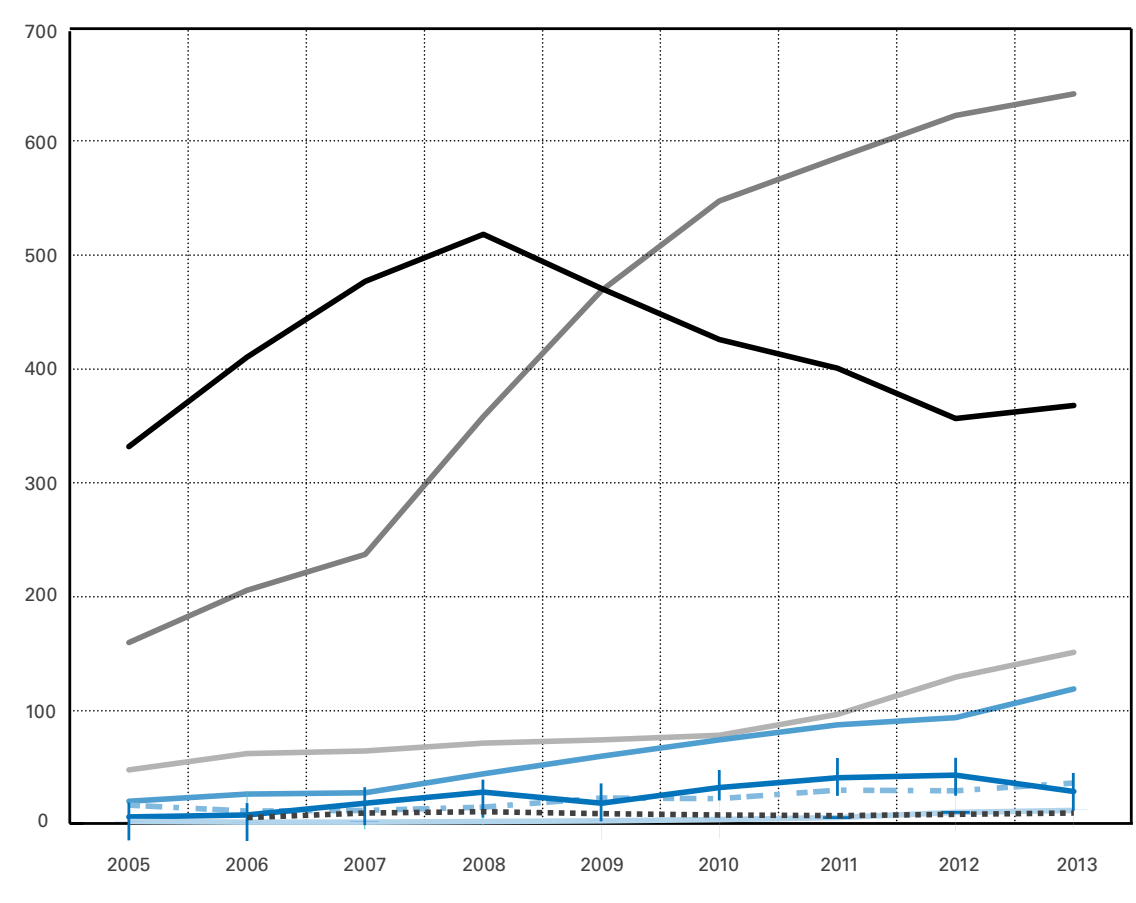

Quelle: Eurostat-Sonderauszüge aus aggregierten AKE-Daten, Version 28.08.2014. Alter: 15-64. Berechnung und Darstellung der Autoren.

Im Prinzip sind Arbeitsmigranten aus EU-Staaten beim Anspruch auf Arbeitslosenunterstützung den einheimischen Bürgern gleichgestellt; in der Praxis jedoch sind sie oft schwächer abgesichert als Einheimische. Dies liegt zum einen daran, dass sie sich ihrer Rechte tendenziell weniger bewusst sind, zum anderen aber auch daran, dass sie häufiger ungeregelte und atypische Beschäftigungen ausüben, die nicht oder nur begrenzt mit Ansprüchen auf Leistungen der Arbeitslosenversicherung verbunden sind (Leschke 2013). Giulietti et al. (2013) stellen fest, dass der Anteil von Zugezogenen, die Sozialleistungen irgendeiner Art beziehen, nur in wenigen Ländern über dem entsprechenden Anteil der Einheimischen lag, und zwar selbst unter Berücksichtigung unterschiedlicher Charakteristika der EU-Migranten. Für Großbritannien befinden Dustmann/ Frattini (2014), dass Einwanderer aus EU-Ländern, die im jeweiligen Untersuchungsjahr des Zeitraums 1995 bis 2011 in Großbritannien ansässig waren, weniger häufig Sozialleistungen (welfare benefits oder tax credits) bezogen und auch seltener in Sozialwohnungen untergebracht waren als Einheimische des jeweiligen Landesteils.

Die oben beschriebenen Trends lassen vermuten, dass sowohl Push- als auch Pull-Faktoren während der 
ABB. 3

\section{Entwicklung der Arbeitslosenquoten für Einheimische (Nat.) und EU10-Bürger in ausgewählten EU15-Ländern, 2007, 2010, 2013}

Angaben in Prozent

2013

2010

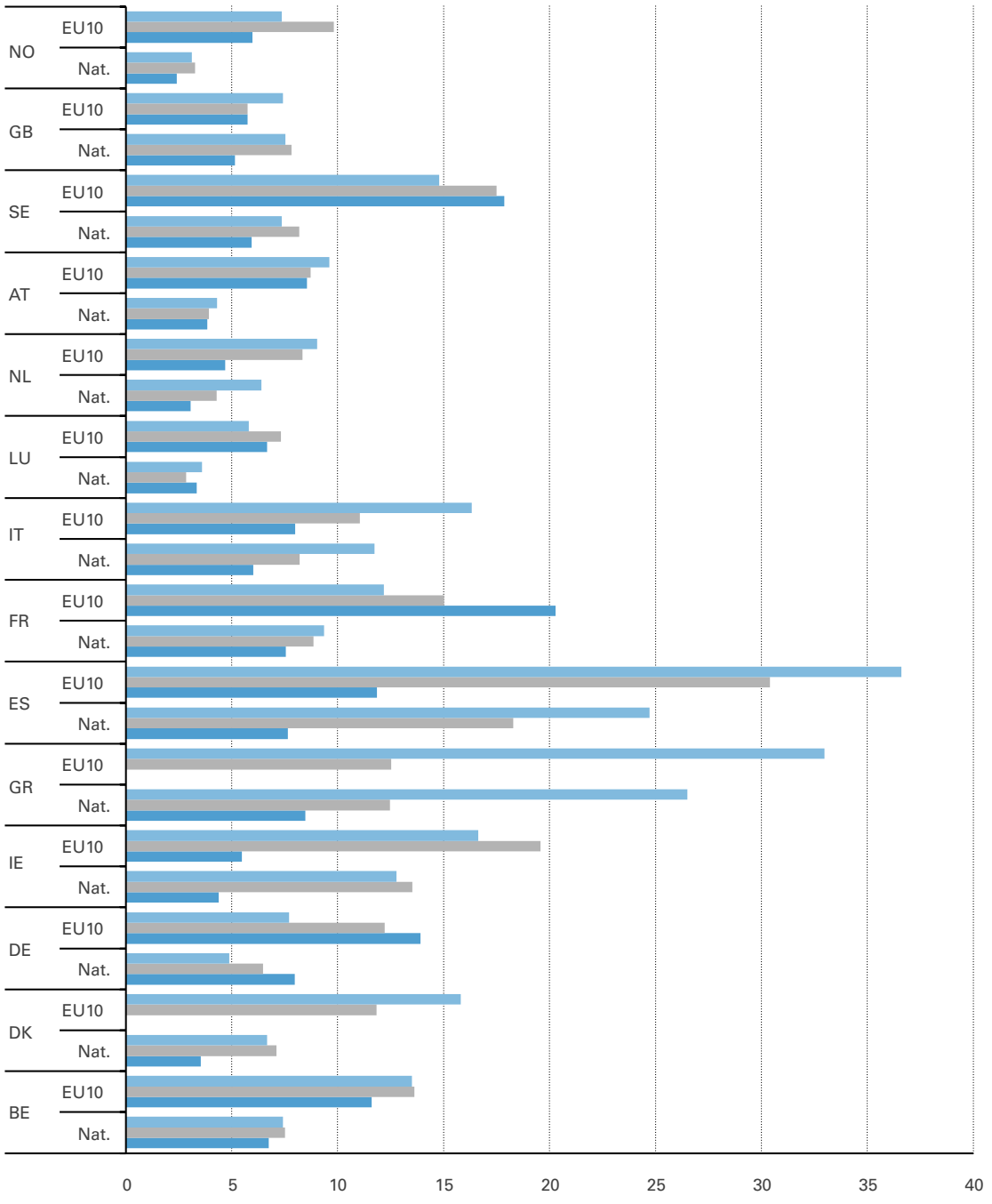

Anmerkung: Daten zu EU10-Staatsangehörigen für bestimmte Länder nicht oder nur unvollständig verfügbar.

Quelle: Europäische Arbeitskräftestichprobe; Berechnung und Darstellung der Autoren. der Arbeitskräftemigration. Für Wanderarbeitnehmer aus anderen Ländern eröffneten sich daheim neue Chancen, die eine Rückkehr ermöglichten - beispielsweise für Migranten aus Polen, wo die Arbeitskräftenachfrage über die Dauer der Krise hinweg vergleichsweise rege war. Ein komplexes Zusammenspiel von Push- und Pull-Faktoren war auch im Fall der Weiterwanderung aus vormals hochattraktiven, inzwischen aber hart von der Krise gezeichneten Zielländern wie Irland zu beobachten: Viele Wanderarbeitnehmer zogen auf der Suche nach günstigeren Arbeitsmarktbedingungen weiter, unter anderem nach Norwegen, wo der Anteil der Migranten aus der EU10 im Zeitraum 2007 bis 2013 um das Zehnfache anwuchs (Europäische Arbeitskräftestichprobe, nicht ausgewiesen; zu Norwegen, vgl. Friberg/Eldring 2013).

Lässt sich also in diesem Zusammenhang überhaupt von Gewinnern und Verlierern sprechen? Einerseits stimmt es natürlich, dass alle Beteiligten von der Freizügigkeit profitieren, da sie mit völlig neuen Chancen verbunden ist. Ebenso stimmt es, dass die Arbeitnehmer, die sich zum risikoreichen Umzug in ein unbekanntes Umfeld durchringen, dies aus freien Stücken und mit der Hoffnung auf ein besseres Leben tun. Da ihre Einkünfte im Zielland trotz aller Schwierigkeiten den vormaligen Verdienst im eigenen Land deutlich übersteigen, darf man sie, kurzfristig betrachtet und im Vergleich zu ihrer früheren Situation, sicher als Gewinner bezeichnen. Die Kardinalfrage lautet jedoch, ob das auch auf längere Sicht stimmt. Um sie zu beantworten, gilt es zunächst, anhand eines Vergleichs der Situation von Wanderarbeitnehmern und einheimischen Bürgern gewisse qualitative Merkmale des Wanderungsgeschehens näher zu untersuchen. Dazu wollen wir uns kurz mit zwei Aspekten befassen: dem Missverhältnis zwischen beruflichen Qualifikationen und Beschäftigungschancen sowie mit der branchenspezifischen Verteilung der Wanderarbeiter aus EU-Ländern im Vergleich zu den Staatsbürgern der Aufnahmeländer. Diese Informationen lassen einige vorläufige Schlüsse zu, ergeben jedoch kein vollständiges Bild. Dazu ist die Frage der Gewinner und Verlierer der EU-Binnenmigration in neuerer Zeit zu komplex und die Gefahr der Vereinfachung zu hoch.

\section{Qualifikations-Mismatch}

Krise dynamischen Veränderungen unterworfen waren. Dies betrifft nicht nur die Wirtschaftslage in den Herkunftsund Zielländern, sondern auch den Wandel der politischen Rahmenbedingungen, zum Beispiel durch Anwendung oder Aufhebung unterschiedlicher Übergangsregelungen in den einzelnen Aufnahmeländern. In einigen Herkunftsländern, beispielsweise Rumänien und Estland, wirkten Push-Faktoren - wie die Auswirkungen der Krise auf die lokalen Arbeitsmärkte oder Einschränkung von Sozialleistungen während der gesamten Krisendauer - als Haupttriebkraft

\subsection{Brain Drain, Brain Gain und Brain Waste im Kontext der EU-Binnenmigration nach der Erweiterung} oft um Qualifikationen und Bildung, doch hängt die Argumentation im Wesentlichen vom jeweiligen Standpunkt ab. Aus der Sicht der Herkunftsländer sorgt sich die wissen-
In der Diskussion über die Arbeitsmigration geht es zwar 
schaftliche Literatur oftmals um den „Brain Drain“, das heißt die übermäßige Abwanderung hochqualifizierter Arbeitnehmer, deren Fachwissen möglicherweise auf dem eigenen Arbeitsmarkt gefragt ist.

Als wichtiges „stilisiertes Faktum“ kann die Tatsache gelten, dass in den EU10-Ländern ein bedeutend höherer Anteil der Erwerbsbevölkerung mittel- und hochqualifiziert ist als in den EU15-Ländern. Der Anteil der Personen, die mindestens die Sekundarstufe II abgeschlossen haben, liegt in der EU10 um fast 20 Prozentpunkte über dem der EU15. Zudem betraf das grenzüberschreitende Migrationsgeschehen seit dem Beitritt der EU10-Länder überwiegend junge Personen, die im Schnitt über ein höheres Bildungsniveau verfügen als ältere. Daraus lässt sich ableiten, dass das Wanderungsgeschehen ab 2004 sich in qualitativer Hinsicht von früheren Migrationswellen unterscheidet (European Integration Consortium 2009).

Angesichts steigender Investitionen in Humanressourcen in der überwiegenden Mehrheit der EU10-Länder, die sich unter anderem durch eine wachsende Studentenzahl im tertiären Bildungsbereich äußern, wurde die Gültigkeit der Brain-Drain-Hypothese für verschiedene EU10-Länder infrage gestellt und vorgeschlagen, das Geschehen als „Brain Overflow" oder Qualifikationsüberschuss zu verstehen, d. h. als Folge eines lokalen Mangels an Beschäftigungsmöglichkeiten, die dem hohen Qualifikationsniveau vor allem jüngerer Bewerber gerecht werden (vgl. z. B. Fihel et al. 2007; Kaczmarczyk/Okólski 2008).

Aus der Perspektive der Aufnahmeländer lautet die Fragestellung: „Brain Gain“ oder „Brain Waste“? „Brain Gain“ liegt vor, wenn Arbeitsmarktengpässe in hochqualifizierten Berufen (z. B. Arztberufen) oder anderen Bereichen (Krankenpfleger, IT-Fachkräfte usw.) durch die Anwerbung von Migranten geschlossen werden. Im Zusammenhang mit der Ost-West-Arbeitskräftemobilität innerhalb der EU haben einschlägige Programme zur Anwerbung hochqualifizierter Arbeitskräfte ebenso eine Rolle gespielt wie Bemühungen, Absolventen aus EU10-Ländern zum Verbleiben im Studienland zu veranlassen, beispielsweise in Deutschland und Österreich. In Großbritannien und Deutschland zielten ähnliche Bemühungen im Rahmen der jeweiligen Übergangsregelungen auch auf Arbeitnehmer aus Rumänien und Bulgarien ab (OECD 2010, S. 42ff.).

„Brain Waste“ bedeutet, dass Migranten in Tätigkeiten beschäftigt sind, deren Anforderungen deutlich unter dem Qualifikationsniveau des Arbeitnehmers liegen. Diese Situation stellten wir auch in einer der Haupt-Schlussfolgerungen einer früheren Untersuchung fest (Galgóczi et al. 2009). Allgemein betrachtet birgt sie die Gefahr, dass kostbare Humanressourcen ungenutzt bleiben; aus der Sicht des einzelnen Arbeitnehmers wiederum entkräftet dieses Missverhältnis die These, dass die Arbeitserfahrung im Ausland das Humankapital eventueller Rückkehrer bereichert. Der Tatbestand widerspricht zudem der Erwartung der Europäischen Kommission (2011a), dass erhöhte Ar- beitsmobilität in Zeiten zunehmender Diversifizierung nationaler Arbeitsmärkte zu einer effizienteren Allokation der Arbeit beitragen wird.

Aus der vorhandenen Literatur lässt sich ableiten, dass auf den Arbeitsmärkten und in den Volkswirtschaften der meisten Herkunfts- und Zielländer im Großen und Ganzen weder „Brain Drain“ noch „Brain Gain“ nachhaltige Spuren hinterlassen werden. Im Falle kleinerer Länder mit starker Abwanderung sowie in gewissen Beschäftigungsbereichen (beispielsweise beim medizinischen Personal) kann sich die Situation jedoch als problematisch erweisen.

Im Folgenden untersuchen wir das Qualifikationsprofil von EU-Wanderarbeitnehmern im Vergleich zu den Qualifikationen der einheimischen Erwerbsbevölkerung für eine Auswahl von EU15-Ländern und besprechen in aller Kürze einige der Befunde, die sich in der akademischen Literatur zum Mismatch zwischen Qualifikation und Beschäftigung finden. Anschließend erhärten wir unsere Argumentation mit Daten zur branchenspezifischen Verteilung der EU-Wanderarbeitnehmer im Vergleich zur einheimischen Erwerbsbevölkerung.

\subsection{Qualifikationsprofil und branchen- spezifische Verteilung}

In der Altersgruppe 15 bis 64 Jahre lassen sich von Land zu Land erhebliche Unterschiede im Qualifikationsprofil sowohl der EU8- und EU2-Migranten als auch der einheimischen Beschäftigten feststellen. Vergleicht man den Qualifikationsstand von Arbeitsmigranten mit dem der Bürger des jeweiligen Ziellandes, kommt man in der Mehrheit der Länder - insbesondere in Frankreich und Großbritannien, etwas weniger deutlich aber auch in Deutschland - für alle drei Gruppen zu sehr ähnlichen Ergebnissen. Lediglich in Spanien finden sich starke Unterschiede zwischen den Profilen von Migranten aus EU8 und EU2 sowie Einheimischen. Insgesamt gesehen scheint es Irland, Großbritannien und Frankreich am besten gelungen zu sein, hochqualifizierte EU10-Arbeitsmigranten anzuwerben. In Italien dagegen ist der Anteil hochqualifizierter Arbeitnehmer aus EU8 und EU2 besonders gering - und unterscheidet sich damit nicht wesentlich von dem Qualifikationsstand der einheimischen Bevölkerung.

Obwohl sich diese Profile oft nicht wesentlich vom Qualifikationsstand der einheimischen Arbeitnehmer unterscheiden, geht aus einer Anzahl jüngerer Untersuchungen hervor, dass ab 2004 aus den neuen Mitgliedstaaten zugezogene Migranten im Schnitt deutlich unter ihrem Qualifikationsniveau beschäftigt sind und ihr Bildungsaufwand sich daher kaum auszahlt (Brain Waste). Das Europäische Integrationskonsortium (2009, S. 97ff.) weist dies für Großbritannien nach, die entsprechenden Kapitel in Kahanec/ Zimmermann (2010) und Galgóczi et al. (2009 und 2012) für eine Reihe weiterer Zielländer. Beispielsweise befindet Bettin (2012, S. 47ff.) anhand von Daten nationaler Arbeitskräfteerhebungen, dass der qualifikatorische Mismatch 
unter Arbeitsmigranten sowohl in Großbritannien als auch in Italien erheblich ist. In beiden Ländern war 2010 ein überproportional hoher Zuwandereranteil auf manuellen Arbeitsplätzen beschäftigt, während einheimische Arbeitnehmer und EU15-Bürger überwiegend in höherqualifizierten Bereichen tätig waren. Die Brain-Waste-Hypothese wird von Dølvik/Eldring (2008, S. 76f.) in Bezug auf Migranten aus den baltischen Staaten und Polen auch für die nordischen Länder bestätigt.

Weitere Hinweise auf das Missverhältnis zwischen Qualifikationsprofil und tatsächlicher Beschäftigung liefert ein Blick auf die branchenspezifische Verteilung von EU8 und EU2-Migranten in den wichtigsten EU15-Zielländern ${ }^{5}$ im Vergleich zur einheimischen Bevölkerung.

In den sechs Wirtschaftssektoren, die für die EU-Arbeitsmigration im Schnitt die größte Rolle spielen - Hotelund Gastgewerbe, Hausarbeit, Verwaltungsdienste und unterstützende Dienstleistungen, Landwirtschaft, Baugewerbe und Industrie - lässt die branchenspezifische Verteilung von EU10-Migranten und Einheimischen sehr unterschiedliche Muster erkennen. Ungeachtet quantitativer Unterschiede gilt für alle der hier betrachteten Zielländer,

\section{ABB. 4}

\section{Qualifikationsstand der Erwerbsbevölkerung: Einheimische, EU8- und EU2-Bürger, 2013}

Angaben in Prozent

- keine Angaben gering mittel $\quad$ hoch

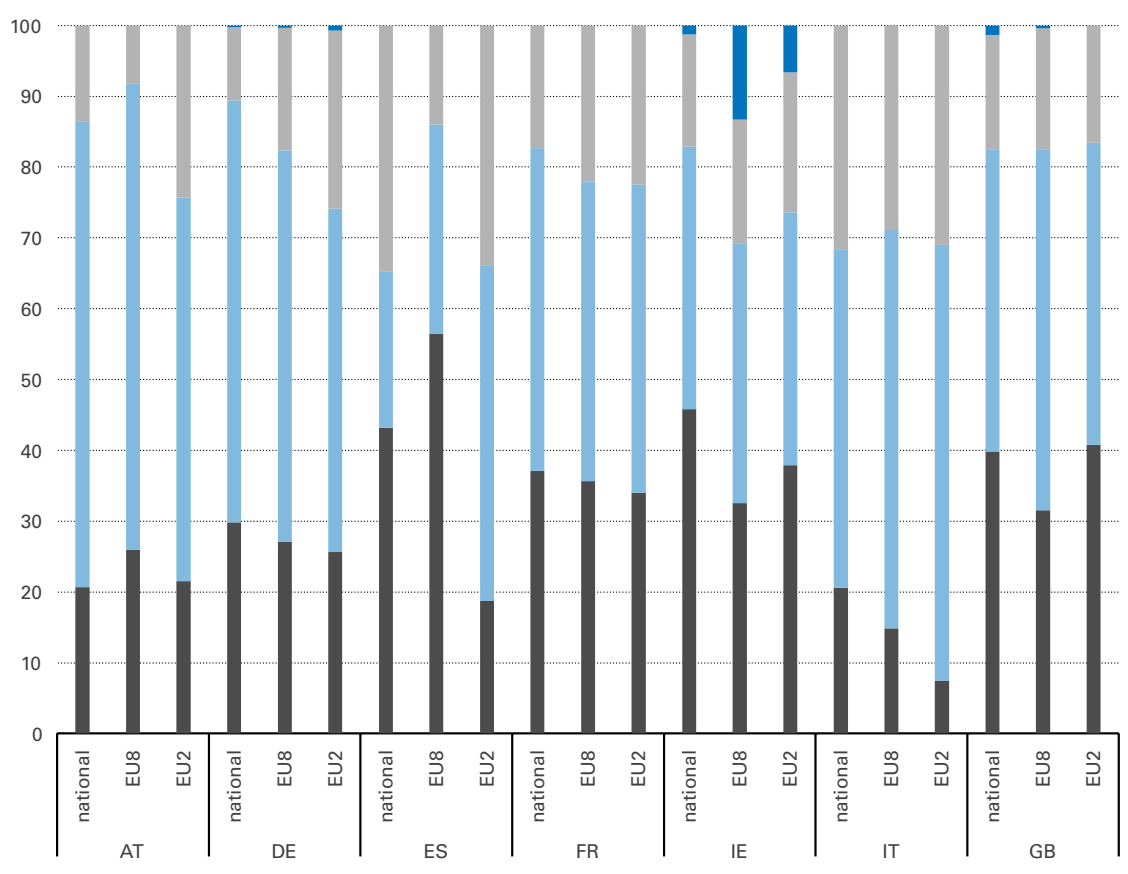

Anmerkung: Dänemark nicht enthalten, da Daten unvollständig

Quelle: Eurostat-Sonderauszüge aus aggregierten AKE-Daten, Version 28.08.2014. Alter: 15-64 Berechnung und Darstellung der Autoren. dass in den Wirtschaftszweigen, in denen Migranten am häufigsten beschäftigt sind, die einheimische Erwerbsbevölkerung unterrepräsentiert ist. In Großbritannien (nicht abgebildet) und Spanien (Abbildung 5) ist dieser Trend am deutlichsten zu erkennen: 70 - 80 \% der EU10-Migranten, aber nur 30-40\% der Einheimischen sind in den sechs ausgewählten Wirtschaftsbranchen beschäftigt. Eine überwältigende Mehrheit der Arbeitsplätze in diesen Bereichen betrifft manuelle Tätigkeiten, die zum allergrößten Teil keine höhere Bildung voraussetzen. Wichtig ist in diesem $\mathrm{Zu}$ sammenhang die Anmerkung, dass die zugrunde gelegten Erhebungsdaten mit großer Wahrscheinlichkeit den Anteil von Arbeitsmigranten in gewissen Bereichen unterschätzen. Dies gilt vor allem für Wirtschaftszweige, in denen Saisonarbeit und die Beschäftigung von Pendlern (z. B. Landwirtschaft) sowie informelle Arbeitsverhältnisse (z. B. Beschäftigung in Privathaushalten) überwiegen.

Abbildung 5 zeigt die relative branchenspezifische Verteilung in Spanien für die Jahre 2008 und 2013, Abbildung 6 die Veränderungen im Zeitraum 2008 bis 2013. Spanien ist in diesem Zusammenhang ein besonders interessanter Fall - sowohl aufgrund seiner früheren Attraktivität für Arbeitsmigranten, insbesondere aus EU2, als auch wegen der katastrophalen Arbeitsmarktlage während der Krise. Die branchenspezifische Verteilung der Beschäftigten aus EU8 und EU2 im Vergleich zu den einheimischen Erwerbstätigen unterscheidet sich nicht wesentlich von der anderer wichtiger EU15-Zielländer: EU10-Migranten sind im Hotelgewerbe, in der Landwirtschaft und als Beschäftigte in Privathaushalten überrepräsentiert. Der Hauptunterschied besteht darin, dass der auf Haushaltshilfen entfallende Anteil der EU10-Zuwanderer in Spanien bedeutend höher liegt (gleiches gilt für Italien, für das keine Daten ausgewiesen sind).

Im Gegensatz zu Deutschland und Großbritannien (nicht ausgewiesen ) hatte die Krise in Spanien sowohl absolut als auch relativ gesehen Einbußen zur Folge (Abbildung 6); zudem waren die krisenbedingten Veränderungen ausgeprägter. Sowohl EU10-Migranten als auch Einheimische sind von hohen Arbeitsplatzverlusten in allen wichtigen Wirtschaftssektoren betroffen; besonders hoch war der Stellenschwund im Baugewerbe und in der Industrie. In den beiden letztgenannten Sektoren waren die Zuwanderer aus der EU10 noch stärker vom Stellenabbau betroffen als die Einheimischen (eine „Lose-lose“-Situation). Für Einwanderer aus der EU10 sind in einigen Sektoren jedoch auch Zugewinne erkennbar. In den Privathaushalten wuchs die Anzahl der EU8-Beschäftigten während der Krise, redu-

5 Wir untersuchten branchenspezifische Daten für Österreich, Dänemark, Deutschland, Italien, Irland, Spanien und Großbritannien (Zahlen auf Anfrage verfügbar). Die generellen Aussagen der folgenden Abschnitte treffen auf alle diese Länder zu. Spezifischere Erkenntnisse stellen wir nur für Spanien vor, da sich dort besonders interessante Entwicklungen abspielen. 
ABB. 5

Verteilung einheimischer, EU8- und EU2Arbeitnehmer auf ausgewählte Branchen in Spanien, 2008 und 2013

Angaben in Prozent
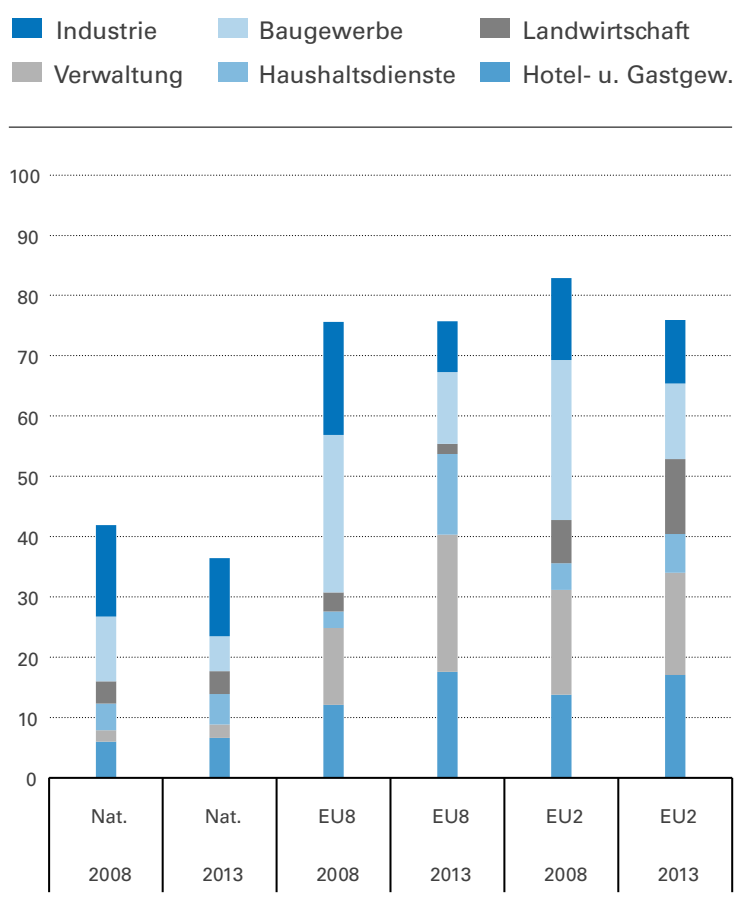

Quelle: Eurostat-Sonderauszüge aus aggregierten AKE-Daten Version 28.08.2014. Alter: 15-64; Berechnung und Darstellung der Autoren.

zierte sich aber gleichzeitig für Personen aus der EU2. In der Landwirtschaft ist das Umgekehrte der Fall, während im Bereich Verwaltungsdienste und unterstützende Dienstleistungen ein starker Anstieg der EU8-Beschäftigung zu verzeichnen war, wobei die absoluten Zahlen allerdings relativ niedrig sind.

Wie im Zusammenhang mit der Untersuchung des Qualifikationsprofils von EU10-Arbeitnehmern in der EU15 angemerkt, liegt das Bildungsniveau der Zuwanderer aus der EU10 deutlich über dem früherer Zuwanderungswellen. Ein charakteristisches Merkmal der EU-Binnenmigration seit 2004 bzw. 2007 scheint jedoch das Missverhältnis zwischen dem Qualifikationsniveau und den ausgeübten Tätigkeiten zu sein; zur Überprüfung dieser These analysierten wir die aktuelle branchenspezifische Verteilung der Arbeitsmigranten - und fanden eine überwältigende Konzentration in Wirtschaftszweigen, die keine höheren Bildungsabschlüsse erfordern.

\section{Schlussfolgerung - Lässt sich überhaupt von Gewinnern und Verlierern sprechen?}

Die Ost-West-Migration im Gefolge der verschiedenen Erweiterungsrunden äußerte sich in verschiedenen Ausprägungen von Migration und Arbeitskräftemobilität, die sich vor dem Hintergrund rapiden wirtschaftlichen und regulatorischen Wandels abspielten. Das Migrationsgeschehen kann folglich nicht losgelöst, sondern nur im Zusammenhang mit verschiedenen Faktoren betrachtet werden. Dazu zählen die von Land zu Land verschiedenen Bemessungen und inhaltliche Ausgestaltung der Übergangsfristen, die unterschiedlich starken Auswirkungen der Krise sowie deren Arbeitsmarktfolgen in den Herkunfts- und den Zielländern.

Die Frage nach den Gewinnern und Verlierern der Arbeitskräftemobilität in der EU drängt sich in diesem $\mathrm{Zu}$ sammenhang geradezu auf. Aufgrund der Komplexität des Sachverhaltes konnten wir jedoch lediglich eine Anzahl von Einflussfaktoren ausmachen, die entscheidend mitbe-

\section{ABB. 6}

\section{Veränderung der Beschäftigungsanteile einheimischer, EU8- und EU2-} Arbeitnehmer in bestimmten Branchen in Spanien, 2008 - 2013

\section{Angaben in Prozent}

national

EU8

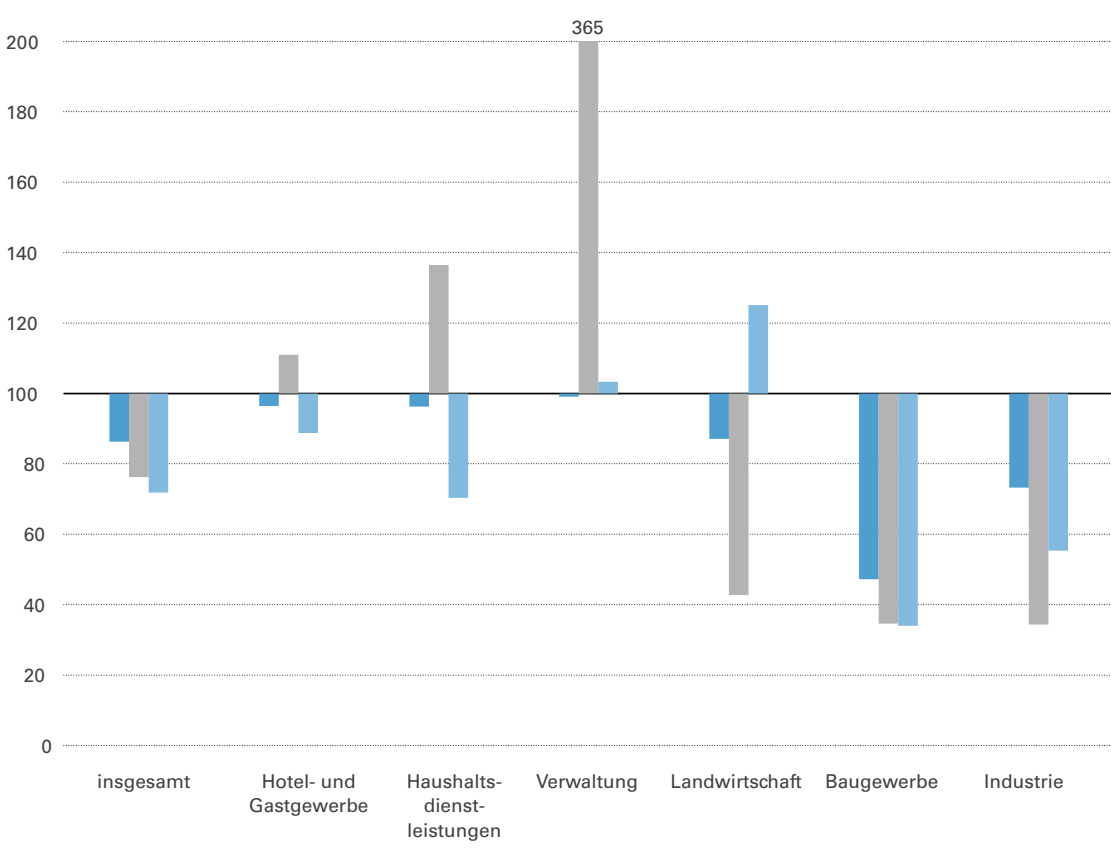

Quelle: Eurostat-Sonderauszüge us aggregierten AKE-Daten, Version 28.08.2014. Alter: 15-64 Berechnung und Darstellung der Autoren. 
stimmt haben, wie sich die Arbeitsmarktschocks jüngerer Zeit auf EU10Migranten und einheimische Arbeitnehmer auswirkten.

Seit den Erweiterungsrunden von 2004 und 2007 ist das Pendel der Push- und Pull-Faktoren, die die Entscheidungen und das Wanderungsverhalten von Migranten beeinflussen, aufgrund rapider und oftmals gegensätzlicher Entwicklungen und Einflüsse nicht zum Stillstand gekommen. Der Prozess der Wirtschafts- und Lohnkonvergenz, der in der Anfangszeit nach dem Beitritt der neuen Länder begann, blieb krisenbedingt stecken. In Bezug auf die Auswirkungen der Krise verläuft die Trennlinie jedoch nicht zwischen Herkunfts- und Aufnahmeländern, sondern zwischen einer besonders stark betroffenen Ländergruppe (vor allem den baltischen Staaten, Spanien und Irland, in der zweiten Phase auch Griechenland und Portugal) und anderen Mitgliedstaaten, die deutlich glimpflicher davonkamen (beispielsweise Deutschland und Polen).

Die heutige Arbeitskräftemobilität innerhalb der EU spricht auf Veränderungen des regulatorischen und volkswirtschaftlichen Umfeldes an. Der Schock der Krise hat die Stellung und die Rolle der Arbeitsmigranten auf den Arbeitsmärkten der Mitgliedstaaten und der Union in den Blickpunkt gerückt. Wenngleich sich die einzelnen Arbeitsmärkte sowohl aufseiten der Herkunfts- als auch der Zielländer sehr unterschiedlich entwickelten, waren Wanderarbeitnehmer stärker als Einheimische von der Krise betroffen, weil die kurzfristige Arbeitskräftemobilität in den meisten Aufnahmeländern zumindest in einem gewissen Umfang als Puffer wirkte. In der Frage nach Gewinnern und Verlierern lässt sich mit Sicherheit sagen, dass Arbeiter in unterwertigen Beschäftigungsverhältnissen als Verlierer bezeichnet werden können. Die Benachteiligten waren in diesem Fall die Arbeitsmigranten, die - oft als Nebeneffekt von Übergangsregelungen - in inoffizielle Arbeitsverhältnisse gedrängt wurden, etwa als Scheinselbstständige oder Entsendearbeitnehmer. Beschäftigungsverhältnisse dieser Art bieten weniger Schutz vor Ausbeutung durch Arbeitgeber oder Arbeitskräfteverleiher und sind mit größerer Unsicherheit und geringeren Sozialleistungsansprüchen verbunden. Unsicherheit war aber auch für reguläre Arbeitsverhältnisse kennzeichnend: Arbeitsmigranten hatten häufiger als Einheimische prekäre Arbeitsverträge. Auf Grund dessen waren EU10-Migranten stärker von Arbeitsmarktspannungen betroffen als die Staatsangehörigen des jeweiligen Aufnahmelandes.

Ein weiteres charakteristisches Merkmal der EU10-Migranten scheint unterwertige Beschäftigung zu sein. Zwar konnten wir uns aufgrund der eingeschränkten Datenverfügbarkeit kein vollständiges Bild machen, doch sprechen Befunde in der akademischen Literatur, Erkenntnisse aus eigenen Untersuchungen sowie die hier vorgestellten branchenspezifischen Verteilungsprofile der EU10-Migranten für die Gültigkeit dieser Aussage. Das ungenutzte Humankapital weist auf eine der größten Herausforderungen hin, mit denen die EU-interne Arbeitskräftemobilität konfrontiert ist, und schadet der gesamten EU. In Anbetracht der Bemühungen auf gemeinschaftlicher und mitgliedsstaatlicher Ebene um eine höhere Effizienz der grenzüberschreitenden Arbeitskräftemobilität lässt sich das Phänomen zudem als ein Scheitern dieser Politik werten.

Insgesamt hat sich die Ost-West-Mobilität in der erweiterten EU somit nicht als Mittel für eine effizientere Allokation der Arbeit auf einem gemeinsamen europäischen Arbeitsmarkt bewährt. Der Beitrag der mobilen Arbeitskräfte zur Arbeitsmarktflexibilisierung hat sich vielmehr als kontrovers erwiesen, nicht nur aus Sicht der Migranten selbst, sondern auch aus Sicht der Zielländer und deren Arbeitsmärkten (man erinnere sich an jüngste Debatten über potenziellen „Sozialleistungsmissbrauch“ u. a. in Großbritannien, Dänemark und Deutschland). Dies gilt es zu beachten, wenn man wie die Europäische Kommission, die verstärkte Arbeitskräftemobilität - einschließlich der Süd-Nord-Migration - innerhalb der EU als zusätzliches Mittel zur Krisenbewältigung betrachtet.

\section{LITERATUR}

Ahearne, A./Brücker, H./Darvas, Z./Weizsäcker, J. von (2009): Cyclical dimensions of labour mobility after EU enlargement, WP 2009/2, Brüssel, http://www.econ.core.hu/file/download/mtdp/MTDP0910.pdf

Ambrosini, J. W./Mayr, K./Peri, G./Radu, D. (2011): The selection of mig rants and returnees: Evidence from Romania and implications: University of California, research paper, Davis, http://www.econ.ucdavis.edu/faculty/gperi/ Papers/return_ro_march_13_2011.pdf

Bettin, G. (2012): Migration from the accession countries to the United Kingdom and Italy: Socio-economic characteristics, skills composition and labour market outcomes, in: Galgóczi, B./Leschke, J./Watt, A. (Hrsg.) (2012): Migration and labour markets in troubled times: Skills mismatch, return migration and policy responses, S. 47-80

Cremers, J. (2011): In search of cheap labour in Europe - Working and living conditions of posted workers, CLR Studies (6), Amsterdam

Dølvik, J-E./Eldring, L. (2008): Mobility of labour from new EU states to the nordic region - Development trends and consequences, Nordic Council of Ministers, Kopenhagen

Dustmann, C./Casanova, M./Fertig, M./Preston, I./Schmidt, C. (2003): The impact of EU enlargement on migration flows, Home Office Report 25/03

Dustmann, C./Frattini, T. (2014): The fiscal effects of immigration to the UK, in:The Economic Journal 124 (580), S. F593-F643

European Commission (2010): The European job mobility action plan 2007-2010, Implementation Report, Brüssel

European Commission (2011a): Employment and social developments in Europe 2011, Brussels

European Commission (2011b): Commission report on transitional arrangements regarding free movement of workers from Bulgaria and Romania, MEMO/ 11/773, Brussels, http://europa.eu/rapid/press-release_MEMO-11-773_en.htm.

European Integration Consortium (2009): Labour mobility within the EU in the context of enlargement and the functioning of the transitional arrangements, Nürnberg

European Labour Force Survey: http://epp.eurostat.ec.europa.eu/portal/ page/portal/labour_market/introduction

Fihel, A./Kaczmarczyk, P./Mackiewicz-Łyziak, J./Okólski, M. (2007):

Labour mobility in the context of enlargement and the functioning of transitional arrangements, Country report: Poland. Centre for Migration Studies, University of Warsaw

Fihel, A./Okólski, M. (2009): Dimensions and effects of labour migration to EU countries:The case of Poland, in: Galgóczi, B./Leschke, J./Watt, A. (Hrsg.): EU labour migration since enlargement: Trends, impacts and policies, Aldershot

Friberg, J. H./EIdring, L. (2013): Labour migrants from Central and Eastern Europe in the Nordic countries : Patterns of migration, working conditions and recruitment practices. Nordic Council of Ministers, Nordic Council of Ministers Secretariat, Kopenhagen

Galgóczi, B./Leschke, J./Watt, A. (Hrsg.) (2009): EU labour migration since enlargement:Trends, impacts and policies, Aldershot

Galgóczi, B./Leschke, J./Watt, A. (Hrsg.) (2012): Migration and labour markets in troubled times: Skills mismatch, return migration and policy responses, Aldershot

Giulietti, C./Guzi, M./Kahanec, M./Zimmermann, K. F. (2013): Unemployment benefits and immigration: Evidence from the EU, in: International Journal of Manpower 34 (1), S. 24-38

Hazans, M. (2012): Selectivity of migrants from Baltic countries before and after enlargement and responses to the crisis, in: Galgóczi, B. et al. (Hrsg.), a. a. O., S. $169-207$

Heinz, F./Ward-Warmedinger, M. (2006): Cross-border labour mobility within an enlarged EU, ECB Occasional Paper Series (52), Frankfurt a. M. Holland, D./Fic, T./Rincon-Asnar, A./Stokes, L./Paluchowski, P. (2011): Labour mobility within the EU -The impact of enlargement and the functioning of the transitional arrangements, final report: European Commission, contract VC/2010/1159 
IDEA Consult ECORYS Netherlands (2011): Study on the economic and social effects associated with the phenomenon of posting of workers in the EU, final report: European Commission, contract VT/2009/062

Kahanec, M./Zimmermann, F. (2010): EU labour markets after post-enlargement migration, Berlin/Heidelberg

Kaczmarczyk, P./Okólski, M. (2008): Demographic and labour-market impacts of migration on Poland, in: Oxford Review of Economic Policy 24 (3), S. $599-624$

Kvist, J. (2004): Does EU Enlargement lead to a race to the bottom? Strategic interaction among EU member states in social policy, in: Journal of European Social Policy 14 (3), S. $301-318$

Leschke, J. (2013): La crise économique a-t-elle accentué la segmentation du marché du travail et de la protection sociale? Une analyse des pays de l'UE (2008-2010), Revue Française des Affaires Sociales (4) : Special Issue on Emplois et statuts atypiques: Quelles protections sociales?

\section{Organisation for Economic Co-operation and Development (OECD)}

(2009): International migration outlook 2009, OECD Publishing

OECD (2010): International migration outlook 2010, OECD Publishing

OECD (2012): International Migration Outlook 2012, OECD Publishing

OECD (2013): International Migration Outlook 2013, OECD Publishing

Stan, S./Erne, R. (2013): Labor history: Explaining Romanian labor migration From development gaps to development trajectories, http://dx.doi.org/10.1080/ 0023656X.2013.843841

\section{AUTOREN}

JANINE LESCHKE, Dr., arbeitet als Außerordentliche Professorin (associate professor) bei der Copenhagen Business School, Department of Business and Politics. Arbeitsschwerpunkte: Arbeitsmarkt und Sozialstaat im europäischen Vergleich.

jle.dbp@cbs.dk

BELA GALGOCZI, Dr., ist Wissenschaftler am Europäischen Gewerkschaftsinstitut (EGI) in Brüssel. Arbeitsschwerpunkte: innergemeinschaftliche Arbeitskräftemobilität, ausländische Direktinvestitionen und Standortwettbewerb, grüneTransformation und Auswirkungen auf die Beschäftigung.

bgalgoczi@etui.org 